\title{
A Propagation Loss Coefficient Model of Low-Frequency Elastic Wave in Coal Strata Set
}

\author{
Yinjing Guo $\mathbb{D}$, Yuanyuan Ju $\left(\mathbb{D}\right.$, Zhen Liu $\mathbb{D}^{D}$, and Jianhua Zhang $\mathbb{D}$ \\ College of Electronic and Information Engineering, Shandong University of Science and Technology, Qingdao, \\ Shandong 266590, China \\ Correspondence should be addressed to Yinjing Guo; gyjlwh@163.com
}

Received 6 November 2019; Revised 21 January 2020; Accepted 8 February 2020; Published 9 March 2020

Academic Editor: Yannis Dimakopoulos

Copyright (c) 2020 Yinjing Guo et al. This is an open access article distributed under the Creative Commons Attribution License, which permits unrestricted use, distribution, and reproduction in any medium, provided the original work is properly cited.

Elastic waves cause energy loss during the transmission of coal measures. These losses include propagation loss, dielectric absorption loss, scattering loss, and frequency migration loss. The absorption loss is mainly caused by the inelastic absorption. The scattering loss is caused by the uneven heat absorption in the formation. The frequency shift loss is caused by the piezoelectric effect of coal-bearing formations and the intermodulation of different frequency signals. After considering the influence factors of the coal seam structure, this paper presents a model of low-frequency elastic waves loss coefficient. The paper proposed the loss coefficient of the elastic wave in the coal measure strata by considering two main attenuation mechanisms: intrinsic absorption and scattering. This paper theoretically studied the effects of the model parameters such as density, porosity, particle size, and wave frequency on the loss of wave energy using COMSOL simulation. Besides, the comparison of MATLAB simulation results shows that the simulation results produced by the model proposed in this paper are similar to the models embedded in COMSOL. This work can be applied to coal, oil, and gas exploration and is also helpful to study the mechanisms of wave attention on the lowfrequency band.

\section{Introduction}

A portion of the energy of an elastic wave will be lost when the elastic wave propagates in a coal medium because of the nonperfect flexible and inhomogeneous characteristics of the medium. The sources of loss include absorption, scattering loss, and frequency migration loss. Determining the generating mechanism of these losses is critical in identifying the elastic wave propagation characteristics in the stratum.

Chirdon et al. propose an elastic wave energy attenuation model to describe these losses in 2006 [1]. They deduced the formula for the wavelet scale domain according to the existing scale energy formula and the propagation equation of a seismic wave of a viscoelastic medium. In some cases, the formula model can be directly used to estimate attenuation factors of the observed seismic reflection data. In other cases, the model is considered overly complicated by some researchers, who instead selected other methods [2-4], such as the control variable method, even though such practices are inadequate at describing the losses. The control variable method has been used to analyze the influence of the frequency, propagation velocity, propagation distance, and quality factor on amplitude loss [5].

Miller et al. focus on the study of wave scattering and attenuation in fiber-reinforced composite laminates. They considered the interfacial damage to wave attenuation. The attenuation due to cracks and frequencies was investigated. It can be shown that a critical rate exists on a given crack size for which the attenuation in the composite medium is at its highest value. Furthermore, the wave attenuation in composite laminates is investigated by incorporating energy transfer onto a layer-wise medium. The overall attenuation coefficient for the laminate is obtained [6]. The ray-tracing method is another method that is often used to simulate the characteristics of elastic wave propagation attenuation and plane elastic waves propagation. Song et al. perform ray tracing based on an accurate near-surface velocity model to extract the signal from shot data and calculate macro- $Q$ value based on the centroid frequency shift method. The 
above two dimensions of the $Q$ value model were merged by information fusion of different scales to build a complete near-surface $Q$ simulation. Finally, prestack seismic data were compensated by way of the absorption attenuation method based on CMP gather, and subsequent processing was performed [7]. The actual numerical application proves the effectiveness of the strategy [8]. Tao presented a wave attenuation regularity equation in detail using the raytracing method [9]. The results showed that the absorption loss of an elastic wave is considerably more significant than the power loss caused by interface reflection on the case of incident angle changes in small increments. The final formula accurately simulated the absorption, but scattering loss was not considered.

In recent years, scholars have conducted in-depth research on the attenuation characteristics of elastic wave propagation $[10,11]$ or the propagation characteristics of seismic waves of elastic media. Many methods reveal part of the elastic wave propagation rules of different research perspectives and provide different respective formula models. We propose another loss coefficient model of a lowfrequency elastic wave of coal measure strata to solve the theoretical problems associated with estimating the propagation loss.

\section{Analysis of Coal Measure Strata Loss}

Coal measure strata are nonperfect elastomers. When an elastic wave is propagating in the layers, the stream will undergo dielectric absorption loss, scattering loss, and frequency transfer loss $[12,13]$. The inelastic level and heat absorption mainly cause absorption loss. Scattering loss results from the nonuniformity of the stratum, and frequency transfer loss is caused by the piezoelectric effect of the layer [14]. To efficiently analyze these losses, we often assume that there is a vibration source of the isotropic coal seam and that an elastic wave propagates in a single direction of the longitudinal wave.

First, we assume that an elastic wave propagates from $r$ to $r+\Delta r$; then, the relative change of an elastic wave energy loss can be written as the following equation:

$$
\frac{E(r+\Delta r)-E(r)}{E(r)}=\left(\eta_{a}+\eta_{s}+\eta_{m}\right) \Delta r,
$$

where $\eta_{a}$ is the absorption loss coefficient, $\eta_{s}$ is the scattering loss coefficient, and $\eta_{m}$ is the frequency transfer loss coefficient; these coefficients are all negative and are in decibels. $\eta=\eta_{a}+\eta_{s}+\eta_{m}$ is a coefficient that represents the characteristic of wave energy loss, and the features of the medium determine it. When $\Delta r \longrightarrow 0$, we obtain the equation

$$
\frac{\mathrm{d} E(r)}{\mathrm{d} r}=\eta E(r) .
$$

Solving this differential equation, we obtain

$$
E(r)=K e^{\eta r}+k_{0} r=K e^{\eta_{a} r} e^{\eta_{s} r} e^{\eta_{m} r},
$$

where $K=E(r) \mid r=0$ denotes the source energy, $\eta$ is the loss coefficient, and $r$ is the propagation distance.
Equation (3) shows that if $\eta$ is a constant, the elastic wave energy will be lost with increasing propagation distance according to the index law. Larger values of $\eta$ and $r$ lead to more significant elastic wave losses in the coal measure strata.

2.1. Absorption. The existing research has shown that absorption loss has the following several influencing factors:

(i) Elastic wave frequency. A higher frequency leads to more significant damage, and vice versa. The elastic waves propagate in the medium and convert mechanical energy into heat energy. A higher rate results in a smaller wavelength, and a lower proportion of medium-sized particles makes it easier to transmit mechanical power to the medium; i.e., $\eta_{a}$ is positively related to the frequency.

(ii) All physical properties of the coal measure strata medium, such as lithology of coal measure strata, elasticity, density, porosity, and viscosity, affect absorption. Flexibility is associated with lower absorption loss. A more compact internal structure leads to lower porosity, and a higher viscosity leads to more significant absorption loss. In other words, $\eta_{a}$ is proportional to the medium density $\rho$, porosity $\varphi$, and thickness $\psi$ but inversely proportional to the penetration $\xi$ [15].

In the space domain, we observe the loss changes in energy $E$ with increasing distance $r$. We select the longitudinal wave as the propagating wave, and $\Delta E$ is the energy change in the wavelength range $\lambda_{p} . \lambda_{p}$ is determined by the circular frequency $\omega$ and velocity $v_{p}$ in the equation $\lambda_{p}=$ $2 \pi v_{p} / \omega[16]$. Thus,

$$
\Delta E=\frac{\mathrm{d} E}{\mathrm{~d} r} \lambda_{p} .
$$

In seismic exploration, we can also use the quality factor $Q$ to study the attenuation of seismic waves, which represents the ratio of the total energy of the elastic wave to the energy loss in a period, which is defined as $Q=2 \pi(E / \Delta E)$ $[17,18]$. Besides, we set the inverse of $Q$ as the specific attenuation $\eta_{a}$, and $\eta_{a}$ is proportional to the medium density $\rho$, porosity $\varphi$, and viscosity $\psi$ but inversely proportional to the penetration $\xi[18,19]$. Thus, we define

$$
Q=2 \pi \frac{E}{\Delta E}=k \rho \frac{\xi}{\psi \varphi^{3}},
$$

where $k$ is a constant. By collating the equation, we obtain

$$
\frac{\mathrm{d} E}{\mathrm{~d} r}=\frac{\omega}{v_{p} Q} E .
$$

Solving equation (6), we obtain

$$
E(r)=E_{0} e^{\omega r / v_{p} Q} .
$$

The propagation velocity of longitudinal waves in the medium is $v_{p}=\sqrt{(\lambda+2 \mu) / \rho}$, where $\lambda$ and $\mu$ are Lame coefficients and $\rho$ is the medium density. Thus, 


$$
\eta_{a}=\frac{\omega}{v_{p} Q}=\frac{2 \pi f}{v_{p} Q}=\frac{2 \pi f}{k \rho v_{p}\left(\xi / \varphi \phi^{3}\right)}=k_{1} \frac{f}{\rho v_{p}\left(\xi / \varphi \phi^{3}\right)} .
$$

Equation (8) represents the absorption loss coefficient of an elastic wave in the coal measure strata, where $k_{1}$ is a constant.

2.2. Scattering Loss. Elastic waves scattering loss occurs when the elastic wave propagates through an inhomogeneous medium [20]. A heterogeneous medium granule with many small interfaces has an uncertain impedance. When an elastic wave propagates through the medium, a portion of the stream will change the path. Along the main direction, the energy of the elastic wave becomes weak; this process is called scattering loss. The method of scattering loss is highly complex because it is related to not only the properties and state of the medium but also the shape, size, and number of solid particles of the medium [21]. In theory, a rough approximation is generally made; that is, nonuniform particles are treated as entirely rigid spherical objects with a radius $R$. If the unit volume contains $n$ scattering particles, we can see that the scattering cross section of a single target of the Rayleigh region is given by the following formula [22]:

$$
\eta_{s}=\frac{4}{\pi} k^{4} V^{2} F^{2} n
$$

where $V$ represents the volume of the goal, $F$ represents the shape coefficient of the goal, and $F$ of the simple geometry can be obtained by looking up the table. Refer to $[23,24]$. The scattering loss coefficient $\eta_{S}$ is

$$
\eta_{s}=\frac{2}{9} \pi k_{1}^{4} R^{6} n,
$$

where $K_{1}$ is the elastic wave number of a $2 \pi$-long unit. The number of particles in the unit volume is $n$. Substituting $K_{1}=(2 \pi / \lambda)$ and $\lambda=\left(v_{p} / f\right)$ into (10), we obtain

$$
\eta_{s}=\frac{32}{9} \frac{\pi^{5} R^{6} f^{4}}{v_{p}^{4}} n
$$

Because the propagation velocity of the longitudinal wave in the medium is $v_{p}=\sqrt{(\lambda+2 \mu) / \rho}$, equation (11) will change to

$$
\eta_{s}=\frac{32 \pi^{5}}{9} \frac{R^{6} f^{4}}{((\lambda+2 \mu) / \rho)^{2}}=k_{2} \frac{R^{6} f^{4}}{((\lambda+2 \mu) / \rho)^{2}} .
$$

In equation (12), which describes the elastic wave scattering loss coefficient in the coal-bearing strata, $K_{2}$ is a constant. The formula indicates that the scattering loss coefficient $\eta_{S}$ is related to the density of the particles of the medium, the radius $R$ of the particles, and the frequency $f$ of the elastic wave.

2.3. Total Loss Coefficient. The frequency migration loss is minimal in the majority of the medium. And it cannot be easily estimated. Therefore, the total loss coefficient of the elastic wave in the coal measure strata can be derived as

$$
\eta=\eta_{a}+\eta_{s}+\eta_{m} \approx k_{1} \frac{f}{\sqrt{(\lambda+2 \mu) / \rho}\left(\xi / \psi \varphi^{3}\right)}+k_{2} \frac{R^{6} f^{4}}{\sqrt{(\lambda+2 \mu) / \rho}^{2}} .
$$

\section{Model Establishment}

China's coalfields are widely distributed and have abundant reserves. Still, but both the coal-bearing rock series (called coal-bearing strata) in China and other countries in the world are composed of coal beds and a set of sedimentary rocks. Coal-bearing levels have a layered structure [25], and the density of coal-bearing levels is less than the thickness of surrounding cliffs. The velocity of elastic waves of coalbearing degrees is lower than that of surrounding rocks [26].

The EDA (Extensively Dilatancy Anisotropy) medium model is a physical expression that is apt to the medium containing cracks (slots). Therefore, the paper considered the anisotropy of the coal seam medium. The coal seam is equivalent to the EDA medium [27]. The model of the coalbearing stratum is shown in Figure 1. The medium parameters are shown in Table 1. It is $2000 \mathrm{~m} / \mathrm{s}$, the shear wave velocity is $1000 \mathrm{~m} / \mathrm{s}$, the density is $1.3 \mathrm{~g} / \mathrm{cm}^{3}$, and the coal seam thickness is $4 \mathrm{~m}$. The upper and lower layers are surrounding rocks, which are isotropic media. The $\mathrm{P}$-wave velocity is $4000 \mathrm{~m} / \mathrm{s}$, the $S$-wave speed is $2000 \mathrm{~m} / \mathrm{s}$, the density is $2.6 \mathrm{~g} / \mathrm{cm}^{3}$, and the thickness is $10 \mathrm{~m}$.

\section{Results and Discussion}

4.1. Loss Model Simulation. COMSOL is based on a finite element algorithm implemented in field simulation software [14].

It has been proven that the model embedded in COMSOL is practical in the transmission attenuation of an elastic wave. We select MATLAB as the simulation platform for simulating the loss formula model proposed above. We also chose the simulation results of the COMSOL model as the comparison standard. By comparing and analyzing two simulation results of COMSOL and MATLAB, the effectiveness of the proposed model is evaluated.

From equations (3) and (13), we obtain

$$
E(r) \approx K e\left(k_{1} \frac{f}{\sqrt{(\lambda+2 \mu) / \rho}\left(\xi / \psi \varphi^{3}\right)}+k_{2} \frac{R^{6} f^{4}}{(\lambda+2 \mu) / \rho^{2}}\right) r+k_{0} r .
$$

Equation (14) is the model simulated by MATLAB. The numerical method is to analyze and solve problems by transforming multiple influencing factors of various singlefactor questions. We set independent $\rho, \xi, \varphi$, and $f$ and dependent variables $E(r)$ in MATLAB. Assume that other influencing factors are constant and change only one of them at a time to study the effect of this modified factor of the characteristics of multi-subchannels in coal-bearing strata. The simulation results are shown in Figures 2-5.

In the COMSOL software, as in MATLAB, the two layers of rock are selected as the top and bottom of the coal seam, and the middle layer is the coal seam. Assume upper and 


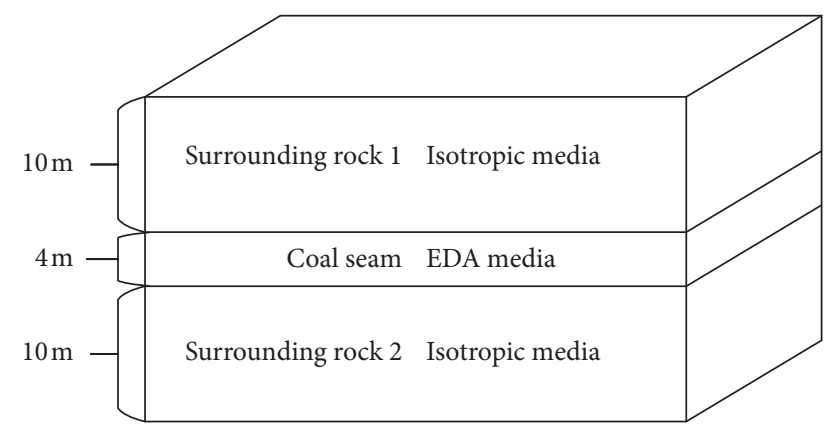

Figure 1: Coal measure strata medium model.

TABle 1: Parameter of the coal stratigraphic media model.

\begin{tabular}{lccc}
\hline Parameter & Surrounding rock 1 & Coal seam & Surrounding rock 2 \\
\hline$v_{p}(\mathrm{~m} / \mathrm{s})$ & 4000 & 2000 & 4000 \\
$v_{s}(\mathrm{~m} / \mathrm{s})$ & 2000 & 1000 & 2000 \\
$\rho\left(\mathrm{g} / \mathrm{cm}^{3}\right)$ & 2.6 & 1.3 & 2.6 \\
$H(\mathrm{~m})$ & 10 & 4 & 10 \\
\hline
\end{tabular}

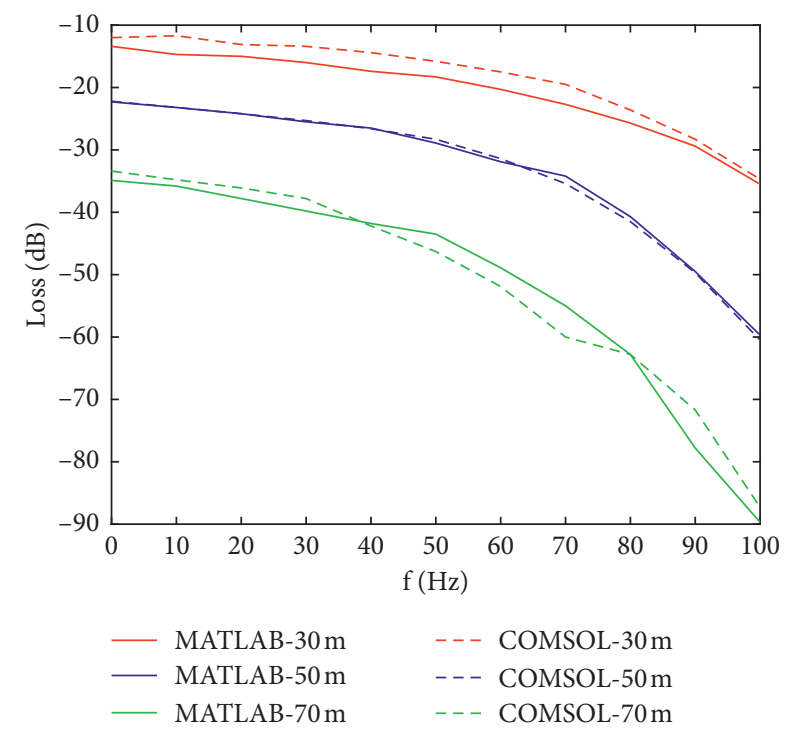

Figure 2: Comparison of loss curves in MATLAB and COMSOL simulations under different frequency effects.

lower surrounding rocks are isotropic media, the simulation length is $200 \mathrm{~m}$, the width of the surrounding rock is $5 \mathrm{~m}$, the thickness of the coal seam is $2 \mathrm{~m}$, and the grid is used to customize the quadrilateral element. The size parameter is the maximum cell size 0.001 , the smallest unit size is $0.001 \mathrm{~m}$, the maximum cell growth rate is 1.3 , and the curvature factor is 0.3 . In the coordinate $(0,0)$, we added the Ricker as the source. Ricker is a kind of seismic wavelet, which is widely used in seismic exploration research $[28,29]$. Formula (15) is the expression of the Ricker, where $f$ is the frequency, and the waveform is shown in Figure 6:

$$
f(t)=\left[1-2(\pi f t)^{2}\right] e^{-(\pi f t)^{2}} .
$$

Near the focal areas and away from the elastic source, three probes are added at distances of 30,50 , and $70 \mathrm{~m}$. The

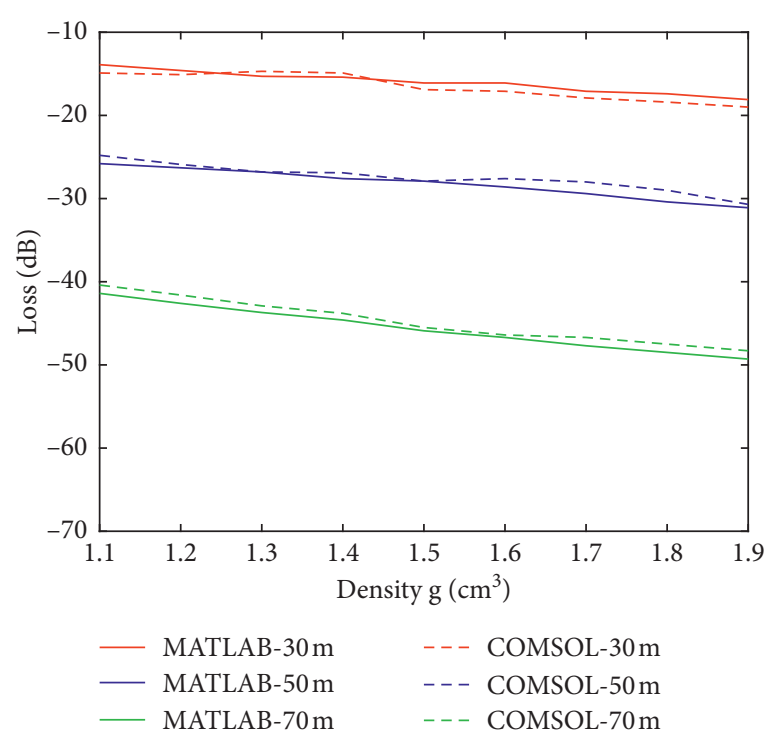

Figure 3: Comparison of loss curves in MATLAB and COMSOL simulations under different density effects.

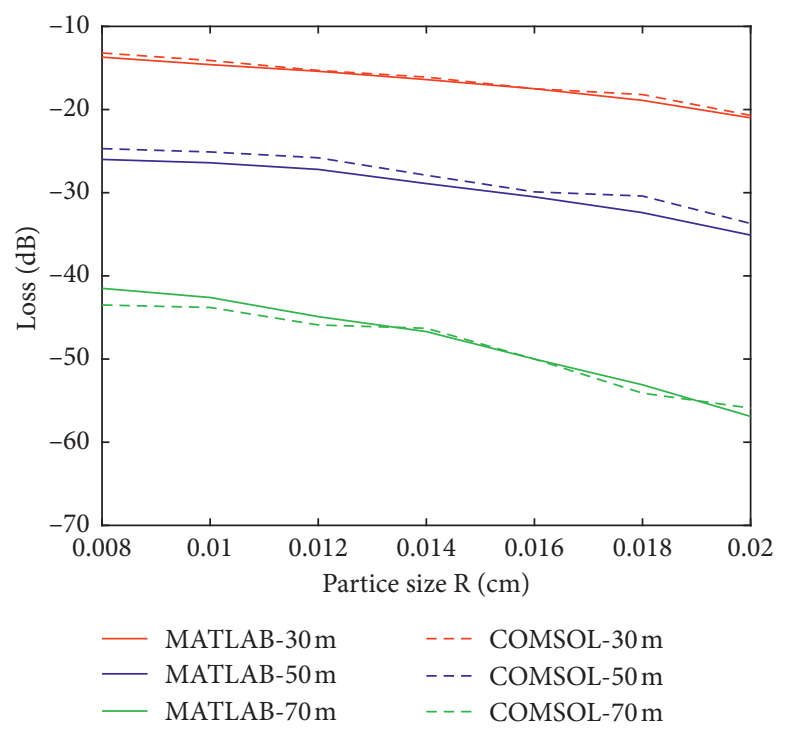

Figure 4: Comparison of loss curves in MATLAB and COMSOL simulations with different particle sizes.

received waveform by the probes is shown in Figure 7 . We can see that the waveform has different degrees of attenuation at 30, 50, and 70 meters. The longer the distance, the smaller the amplitude, and the more significant the attenuation.

4.2. Effect of Frequency on Loss. The relationship between the loss and rate for a medium density of $\rho=1.4^{\circ} \mathrm{g} / \mathrm{cm}^{3}$, a particle size of $R=0.014 \mathrm{~cm}$, the porosity of $\varphi=0.25$, and a frequency range of $0.01-100 \mathrm{~Hz}$ is shown in Figure 2.

Figure 2 illustrates that the two simulation results to exhibit the same variation tendency towards the relationship between loss and frequency. Still, the loss of our model is less 


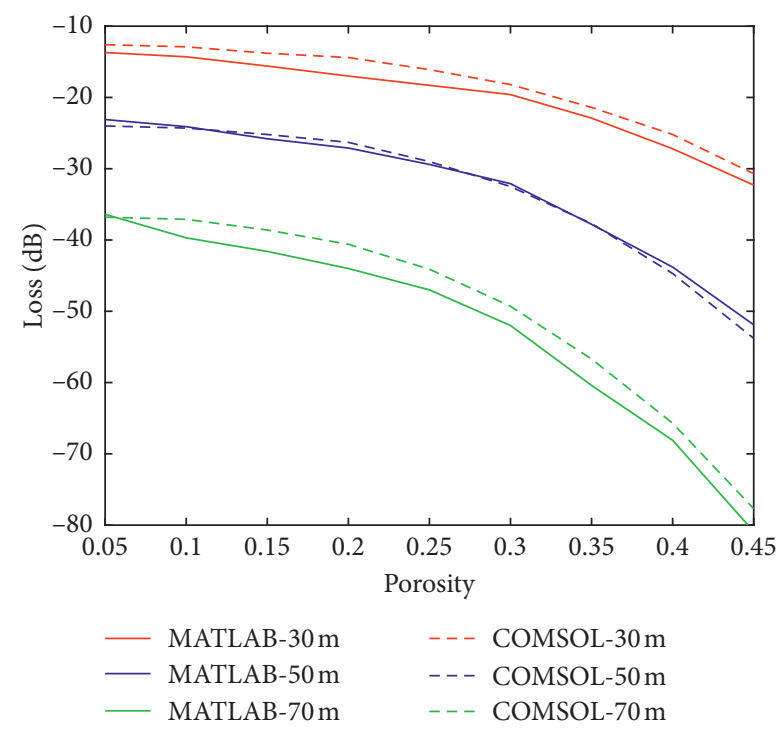

FIGURE 5: Comparison of loss curves in MATLAB and COMSOL simulations with different porosities.

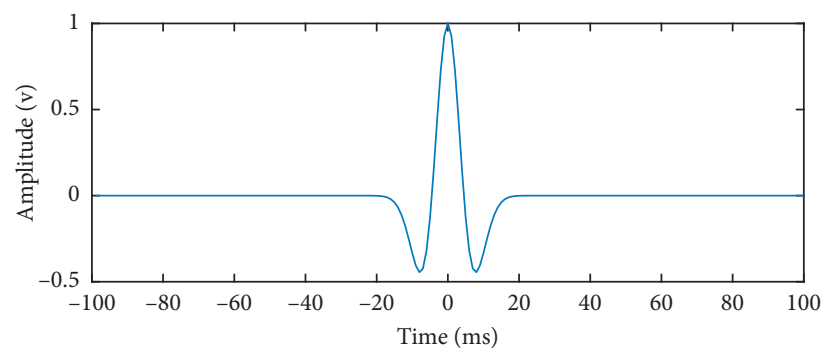

FIgURE 6: Ricker waveform.

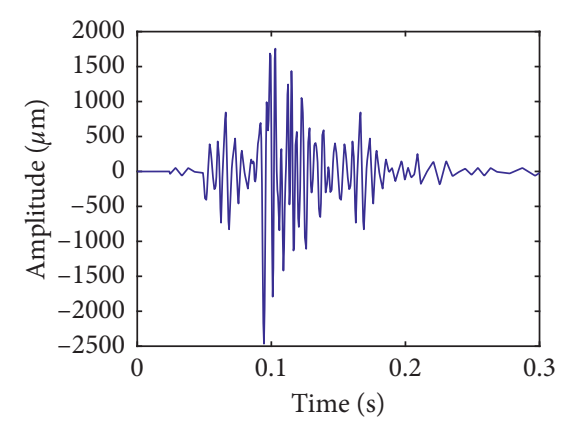

(a)

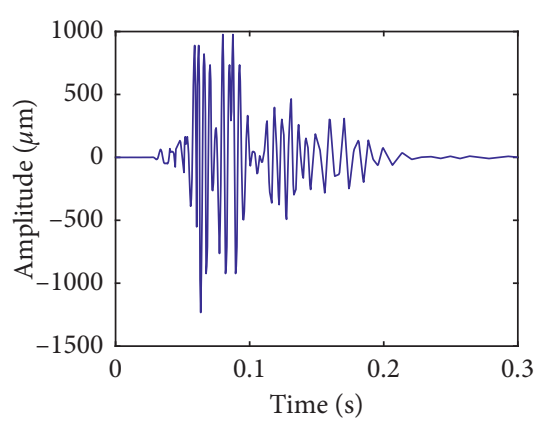

(b)

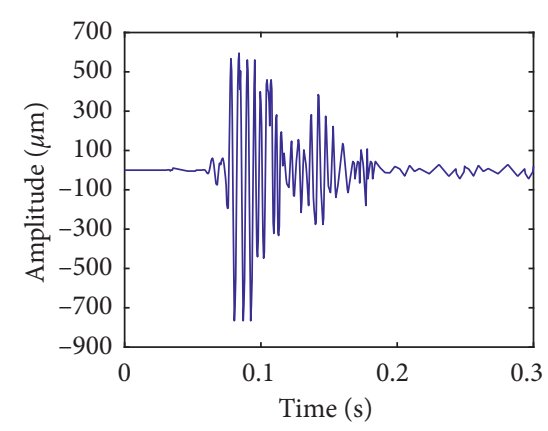

(c)

Figure 7: The received waveforms by the probes at distances of (a) $30^{\circ} \mathrm{m}$, (b) $50^{\circ} \mathrm{m}$, and (c) $70^{\circ} \mathrm{m}$.

than that of COMSOL. At rates of $0-60 \mathrm{~Hz}$, the loss has a weak dependence on frequency, and at frequencies above $60 \mathrm{~Hz}$, the energy loss increases rapidly with increasing frequency. In other words, $60 \mathrm{~Hz}$ is a fundamental frequency in the elastic wave propagation simulation.

4.3. Effect of Density on Loss. We select $60 \mathrm{~Hz}$ as the simulation frequency, $R=0.014 \mathrm{~cm}$ as the particle size, and $\varphi=$ 0.25 as the porosity. Different coal seam densities are chosen to evaluate the model, with values in the range of $1.1-1.9 \mathrm{~g} / \mathrm{cm}^{3}$. The simulation results of the relationship between the loss and coal density are shown in Figure 3.

Figure 3 illustrates that the loss increases gradually with increase in the density of the coal seam, and the maximum value of the loss exceeds $50 \mathrm{~dB}$. The increase in loss is relatively smooth, with increase in the mass of the medium; the relationship can be approximated as linear to a frequency of $60 \mathrm{~Hz}$. The simulation result is similar to that obtained with COMSOL, but our simulation curve is smoother. 
4.4. Effect of Particle Size on Loss. The relationship between loss and particle size for a medium density of $\rho=1.4 \mathrm{~g} / \mathrm{cm}^{3}$, a frequency of $60 \mathrm{~Hz}$, a porosity of $\varphi=0.25$, and particle size ranging from 0.008 to $0.02 \mathrm{~cm}$ is shown in Figure 4 .

Figure 4 illustrates that the loss increases gradually with increasing particle size. When the particles are more massive than $0.014 \mathrm{~cm}$, the loss changes more rapidly, with a maximum loss value of $55 \mathrm{~dB}$; thus, the particle size influences the amount of loss, and our model simulates a smaller loss than COMSOL because of the particle size.

4.5. Effect of Porosity on Loss. We simulated the relationship between the loss and porosity for a medium density of $\rho=1.4 \mathrm{~g} / \mathrm{cm}^{3}$, a frequency of $60 \mathrm{~Hz}$, a particle size of $R=0.014 \mathrm{~cm}$, and a porosity ranging from 0.05 to 0.45 .

As is shown in Figure 5, the elastic wave loss and porosity are positively correlated, and the loss rises with the increase in porosity. The loss increases relatively slowly when the porosity changes from 0.05 to 0.25 , whereas the loss rises rapidly when the porosity is higher than 0.25 . The maximum loss is approximately $80 \mathrm{~dB}$.

Figures 2-5 illustrate that the simulation curves obtained with our model have the same change tendency as those obtained with COMSOL. The model embedded in COMSOL includes more inference factors that affect wave energy loss. Thus, the loss value obtained with COMSOL is slightly higher, and the loss curve is not as smooth because the propagation of the wave in real coal measure strata is highly complex.

\section{Conclusions}

Because it is challenging to simulate various forms of coal seams, the elastic wave energy loss formula model proposed to this paper is only an approximate value. However, when only one loss factor is considered, the proposed model improves the existing model. A comparison of the simulation results from MATLAB and COMSOL demonstrated that our model provides results that are highly similar to the results obtained with COMSOL; however, some differences existed. The following conclusions are drawn from this study:

(1) Although the proposed model only considered two principal loss coefficients, the simulation result is nearly identical to that obtained with COMSOL, with simulation errors of less than $3 \mathrm{~dB}$ between the two models. The energy losses in the COMSOL result are always higher because the COMSOL models include more energy loss factors. The model proposed in this paper is valid based on the comparison of COMSOL.

(2) Considering various types of media in coal measure strata, the loss of an elastic wave increases with the increase in frequency, density, particle size, or porosity. The loss curve has an inflection point when the frequency is $60 \mathrm{~Hz}$, the porosity is 0.25 , and the rate of loss increases rapidly when the frequency is greater than $60 \mathrm{~Hz}$, or the porosity is higher than 0.25 .
(3) In nature, many factors influence the loss characteristics of an elastic wave in coal measure strata because the coal-bearing strata of the internal structure are highly complex. The surrounding rock pressure and confining pressure, the coal seam thickness, and the mineral component of the medium all affect the loss characteristics of an elastic wave; however, the formula model proposed in this paper only includes the effects of the frequency, density, particle size, and porosity. As a result, this model has several limitations.

Also, the model is not comprehensive in practical applications. Although more research work is required to improve the model, it is still beneficial for analyzing the propagation energy loss of a low-frequency elastic wave according to a single influence factor. The COMSOL software simulation indicated that the grid division and step size selection would affect the accuracy of the calculation results; however, the accuracy is adequate for evaluating the model. Further research on the model will focus on in situ measurements and additional factors influencing the propagation energy loss.

\section{Data Availability}

All data included in this study are available upon request by contact with the corresponding author.

\section{Conflicts of Interest}

The authors declare that there are no conflicts of interest regarding the publication of this paper.

\section{Acknowledgments}

This paper was funded by the National Natural Science Foundation of China (Grant no. 61471224) and Shandong Province Key R\&D Project (Grant no. 2018GHY115022).

\section{References}

[1] D. Chirdon, T. Barkand, N. Damiano et al., Emergency Communication and Tracking Committee Underground Communication and Tracking Systems Tests at Consol Energy Inc, McElroy Mine, The United States Mine Safety and Health Administration, Crystal City, VA, USA, 2006.

[2] I. L. S. Braga and F. S. Moraes, "High-resolution gathers by inverse Q filtering in the wavelet domain," Geophysics, vol. 78, no. 2, pp. V53-V61, 2013.

[3] G. Wang, R. Wang, T. Li et al., "Numerical-model of pore structure effects on acoustic attenuation in dry rocks," Process in Ucophysics, vol. 29, no. 6, pp. 2766-2773, 2014.

[4] K. Asamcnc, L. Hudson, and M. Sundaresan, "Influence of attenuation on acoustic emission signals in carbon fiber reinforced polymer panels," Ultrasonics, vol. 59, pp. 86-93, 2015.

[5] S. C. Gupta and A. Kumar, "Seismic wave attenuation characteristics of three Indian regions: a comparative study," Current Science, vol. 82, no. 4, pp. 407-413, 2002.

[6] D. Miller, S. Das, X. Zhou, and A. Chattopadhyay, "Elastic wave attenuation in composite laminates with cracks," in 
Proceedings of Smart Structures and Materials 2005-Smart Structures and Integrated Systems, SPIE, San Diego, CA, USA, March 2005.

[7] F. Zhu, F. U. Ying-Lu, S. F. Zhang, W. MU, and M. K. Cao, "A review of research on the mechanism of attenuation and energy compensation," Progress in Geophysics, vol. 22, no. 4, pp. 1147-1152, 2007.

[8] J. J. Song, J. Y. Yu, M. Zhang, and C. Y. Yu, “A new strategy for absorption attenuation compensation in the near-surface medium," in Proceedings of SEG International Exposition and 86th Annual Meeting, SEG, Lobby, DC, USA, October 2016.

[9] J. Tao, "Discussion on some questions about through earth radio communication system," Journal of Taiyuan University of Technology, vol. 31, no. 6, pp. 690-693, 2000.

[10] X. Liu, J. Cui, X. Li, and Z. Liu, "Study on the attenuation characteristics of elastic waves in different types of rocks," Chinese Journal of Rock Mechanics and Engineering, vol. 37, no. S1, pp. 3223-3230, 2018.

[11] Y. Zhao, T. Yang, F. Xiao, P. Zhang, Q. Yu, and G. Liu, "Analysis of propagation attenuation characteristics of elastic waves in medium-grain sandstone," Journal of Vibration, Measurement \& Diagnosis, vol. 38, no. 2, pp. 285-291, 2018.

[12] H. Zhou and L. Fu, "Scattering and intrinsic absorption characteristics of spectral attenuation in ultrasonic experiments," Chinese Journal of Geophysics, vol. 61, no. 3, pp. 1083-1094, 2018.

[13] I. D. Gupta and M. D. Trifunac, "Attenuation of strong earthquake ground motion-II: dependence on geology along the wave paths from the Burmese subduction zone to northeastern India," Soil Dynamics and Earthquake Engineering, vol. 112, pp. 256-276, 2018.

[14] N. J. Watson, T. Hazlehurst, M. J. W. Povey, A. Drennan, and P. Seaman, "COMSOL modelling of the acoustoelastic effect," Journal of Physics: Conference Series, vol. 581, p. 012008, 2015.

[15] R. L. Zhang and H. L. Wang, "Experimental study on the relationship between the solidity coefficient of coal and porosity," Coal Mine Safety, vol. 45, no. 10, pp. 1-3, 2014.

[16] J. Hao, Y. Zhao, and Y. Guo, "Transmission loss characteristics of plane elastic wave signal in stratum medium," Journal of Coal, vol. 36, no. S1, pp. 211-214, 2011.

[17] J. Zhao, "Estimation of quality factor Q from pre-stack CMP records," Acta Geophysica Sinica, vol. 56, no. 7, pp. 24132428, 2013.

[18] T. Gong, Study on Attenuation Law of Near-Surface Seismic Waves and Extraction of Quality Factors, China University of Petroleum, Beijing, China, 2010.

[19] Q. Zhao, Study on Seismic Wave Absorption Attenuation Characteristics and Quality Factor Q Inversion Methods and Applications in Near-Surface Formations, Henan Polytechnic University, Jiaozuo, China, 2018.

[20] Z. Hu, J. Fan, Z. P. Zhen, and W. Y. Shuang, "Acoustic scattering from elastic target buried in water-sand sediment," Acta Physica Sinica, vol. 65, p. 064301, 2016.

[21] A. Pirogova, B. Gurevich, R. Pevzner, and S. Vlasov, "Study of intrinsic versus scattering attenuation of seismic waves from borehole measurements," in Proceedings of 88th Society of Exploration Geophysicists International Exposition and Annual Meeting SEG, Anaheim, CA, USA, October 2018.

[22] G. Wu, W. Deng, and J. Jiang, "Research on Rayleigh Zone division and scattering characteristics of radar target," Journal of Radio Science, vol. 22, no. 3, pp. 476-480, 2007.

[23] J. C. Stover, S. Schroeder, C. Staats, V. Lopushenko, and E. Church, "Linking Rayleigh-Rice theory with near-linear shift-invariance in light scattering phenomena," in
Proceedings of Reflection, Scattering, and Diffraction from Surfaces V, SPIE, San Diego, CA, USA, October 2016.

[24] P. Huang and H. Yin, Radar Target Characteristics, Electronic Industry Press, Beijing, China, 2005.

[25] A. Li, Research on Key Technologies of Elastic Wave Penetrating Communication Channel Detection, Shandong University of Science and Technology, Shandong, China, 2010.

[26] T. Liu, Slot Wave Seismic Exploration, China University of Mining and Technology Press, Beijing, China, 1994.

[27] X. He, Z. Zhu, L. Peng, and G. Lu, "Propagation characteristics of seismic waves in viscoelastic EDA media," Progress in Geophysics, vol. 32, no. 1, pp. 363-370, 2017.

[28] G. Wang, Y. Guo, S. Hu et al., "Effects of velocity dispersion effect and absorption effect on ricker wavelet waveform," Mining Research and Development, vol. 34, no. 2, pp. 26-29, 2014.

[29] Y. Zhang, Y. Wan, Y. Shi et al., "Research on the frequency of lake wavelets," Progress in Geophysics, vol. 32, no. 5, pp. 2162-2167, 2017. 